\title{
Tuning the magnetic properties of permalloy-based magnetoplasmonic crystals for sensor applications
}

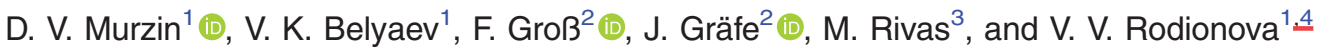 \\ ${ }^{1}$ Immanuel Kant Baltic Federal University, Kaliningrad, 236041, Russia \\ ${ }^{2}$ Max Planck Institute for Intelligent Systems, Stuttgart, 70569, Germany \\ ${ }_{3}^{3}$ Universidad de Oviedo, Oviedo, 33003 , Spain \\ ${ }^{4}$ National University of Science and Technology, MISiS, Moscow, 119049, Russia \\ *E-mail: dvmurzin@yandex.ru
}

Received August 16, 2019; revised October 29, 2019; accepted December 3, 2019; published online MM DD, 2019

\section{Introduction}

Recent rapid advancements in nanomaterial development have had a huge impact on the elaboration of different devices, including magnetic field sensors. Nowadays, magnetic field sensors are being used in orientation devices, ${ }^{1,2}$ ) control $^{3,4)}$ and security ${ }^{5,6)}$ systems, medicine, ${ }^{7,8)}$ space exploration $^{9,10)}$ and other noteworthy applications. Depending on the application, magnetic field sensors have different linear dimensions and levels of accuracy, efficiency, locality and sensitivity. However, the modern tendency for miniaturisation of devices has led to a stronger necessity for highly localised and sensitive magnetic field sensors.

Nowadays, the most sensitive and widely used magnetic field sensors are based on the Hall effect ${ }^{11,12)}$ and on superconducting quantum interference devices. ${ }^{13,14)}$ However, the sensitivity of these devices drops with a decrease of the probe area. $^{15,16)}$ Another possible way to design magnetic field sensors neglecting the influence of linear dimensions on the sensitivity and locality of the device is based on the use of magneto-optical effects in Voigt geometry. ${ }^{17-19)}$ The magnitude of magneto-optical response is usually very weak even for ferromagnetic materials, but it can be enhanced by the use of magnetoplasmonic crystals (MPICs). ${ }^{20,21)}$ MPICs are periodically nanostructured ferromagnetic surfaces ${ }^{22,23)}$ that, due to diffraction effects, support the excitation and propagation of surface resonant evanescent coupled oscillations of metallic plasma and photons called surface plasmon polaritons. ${ }^{24,25)}$ This method allows one to measure precisely the external magnetic field in the area limited only by the size of the optical spot and the penetration depth of light. Recently, a prototype of permalloy-based magnetic field sensor has achieved sensitivity as high as $4.4 \times 10^{-6}$ Oe in a modulating alternating field of 5.0 Oe at an area of $1 \mathrm{~mm}^{26)}$ According to theoretical calculations, its sensitivity can be tuned up to $10^{-7} \mathrm{Oe}^{27)}$ which is appropriate for biomedical applications.

It has been proved that the magneto-optical response of MPIC structures strongly correlates with their magnetic properties, which in turn allows the tuning of the magnetic field sensor's

parameters. $^{26,28,29)}$

This

fact opens up a way to improve the sensitivity of MPlC-based magnetic field sensors by noise reduction and amplification of magneto-optical response by varying the MPIC's parameters such as the ferromagnetic layer thickness and the diffraction grating period and height.

In our previous work ${ }^{26)}$ estimations of the signal-to-noise ratio were done to calculate sensitivity, the measurable field region and the required modulating $\mathrm{AC}$ magnetic field for sensing applications. It was found that an increase of the ferromagnetic layer thickness leads to the occurrence of a stepped behaviour of the field-dependent magneto-optical response and the decrease of signal-to-noise ratio values. In this paper, an explanation of such stepped behaviour is shown. A way to tune the coercive force and effective anisotropy of MPICs by changes in the ferromagnetic layer thickness and spatial parameters of the substrates is revealed.

\section{Experimental methods}

MPICs were fabricated using the DC magnetron sputtering technique. Two series of samples were made with the deposition of $100 \mathrm{~nm}$ or $130 \mathrm{~nm}$ permalloy $\left(\mathrm{Ni}_{80} \mathrm{Fe}_{20}\right.$ or Py) onto smooth planar silicon substrates, $\mathrm{Sub}_{1}$, and polymer substrates, $\mathrm{Sub}_{2}$ and $\mathrm{Sub}_{3}$, with quasi-sinusoidal and trapezoidal diffraction gratings, respectively. The spatial parameters of the gratings are as follows: periods $d_{2}=320 \mathrm{~nm}$ and $d_{3}=740 \mathrm{~nm}$ and profile heights $h_{2}=20 \mathrm{~nm}$ and $h_{3}=100 \mathrm{~nm}$ for $\mathrm{Sub}_{2}$ and $\mathrm{Sub}_{3}$ respectively. The deposition process was carried out at a temperature below $100{ }^{\circ} \mathrm{C}$, constant pressure of 3 mTorr, constant argon flow equal to $10 \mathrm{ccm}$ and constant power of $50 \mathrm{~W}$, to prevent heat deformation of the polymer substrates. Images of the fabricated MPICs' surfaces based on $\mathrm{Sub}_{2}$ and $\mathrm{Sub}_{3}$ were obtained by an atomic force microscope, and are shown in Fig. 1. The obtained images show that the nanostructures' height and periodicity errors were in the vicilitieyMAP0C6\% $\%$ avere found to have a strong in-plane anisotropy that formed an easy magnetisation axis (EMA), directed along the stripes of the diffraction gratings, and a hard 

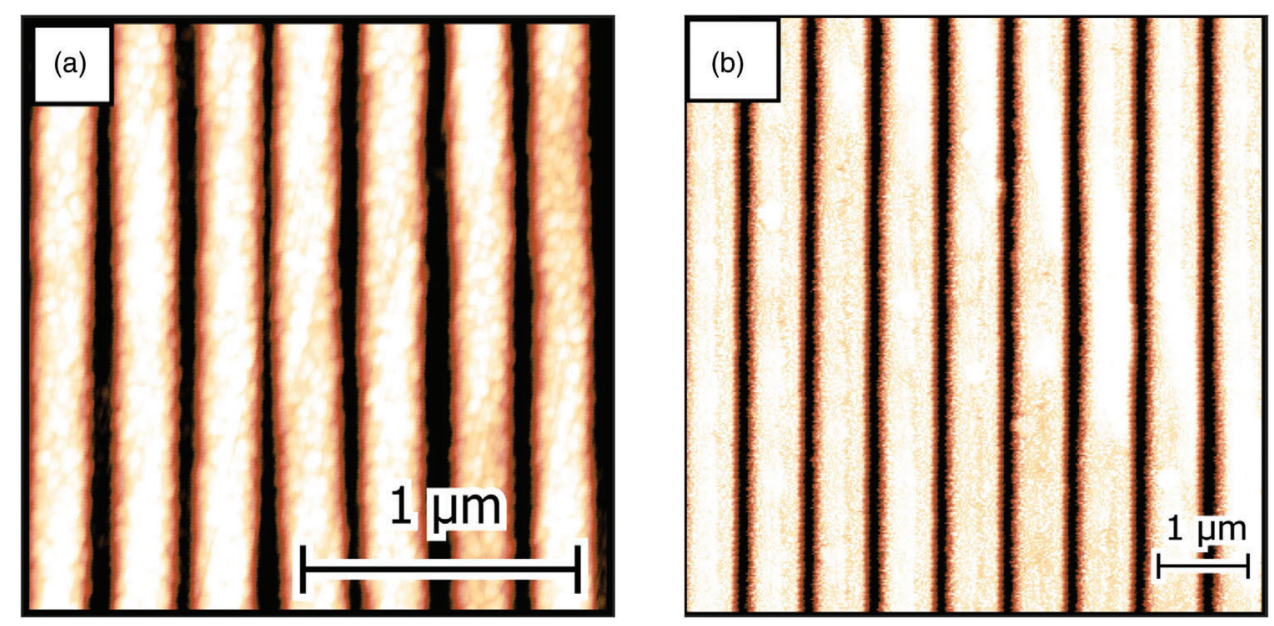

Fig. 1. (Colour online) Atomic force microscope images of MPICs' surfaces based on a) $\mathrm{Sub}_{2}$ and b) $\mathrm{Sub}_{3}$.

magnetisation axis, perpendicular to the stripes. ${ }^{30,31)}$ Also, the MPICs based on the $\mathrm{Sub}_{2}$ and $\mathrm{Sub}_{3}$ substrates had different anisotropy coefficients in regions with tilted and bent parts due to their morphological features. ${ }^{32)}$ The films deposited onto $\mathrm{Sub}_{1}$ were magnetically isotropic. ${ }^{30,31)}$ Measurements of the integral and local magnetic properties in an external magnetic field along the EMA were done with a vibrating-sample magnetometer (VSM) and with a NanoMOKE III magneto-optical magnetometer in the longitudinal magneto-optical Kerr effect (LMOKE) geometry, respectively. Also, the integral magnetic properties for the $\mathrm{Sub}_{3}$-based MPICs were measured in-plane along the EMA, out-of-plane and additionally at $45^{\circ}$ relative to the plane. All measurements were done at room temperature. To analyse the magnetisation reversal processes of the MPICs, the differential susceptibility was calculated as the first-order derivative of the magnetisation with respect to the applied field in hysteresis loops, $\chi_{d}=d M / d H . \quad \chi_{d}$ also represents the switching field distribution (SFD). The FWHM for the calculated $\chi_{d} / \chi_{d}(\max )$ was estimated to determine the (a) $\mathrm{Sub}_{1}$
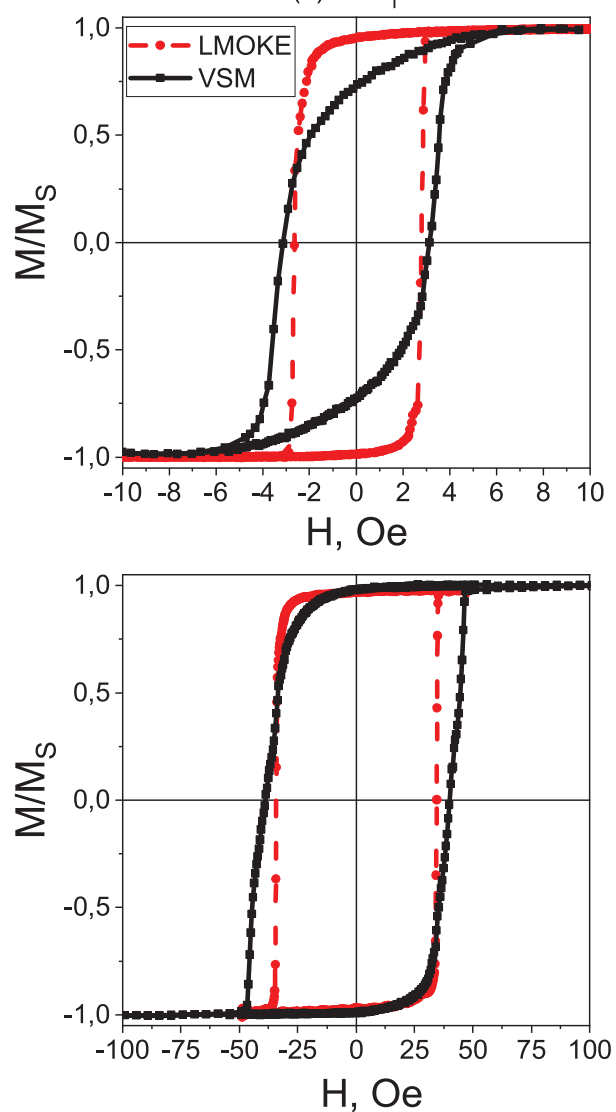

(b) $\mathrm{Sub}_{2}$
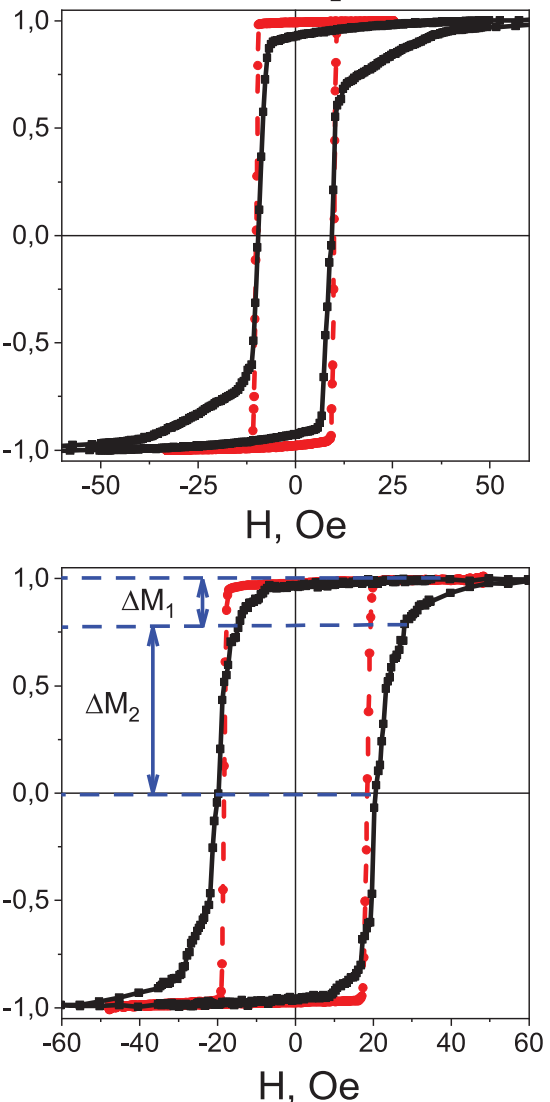

(c) $\mathrm{Sub}_{3}$
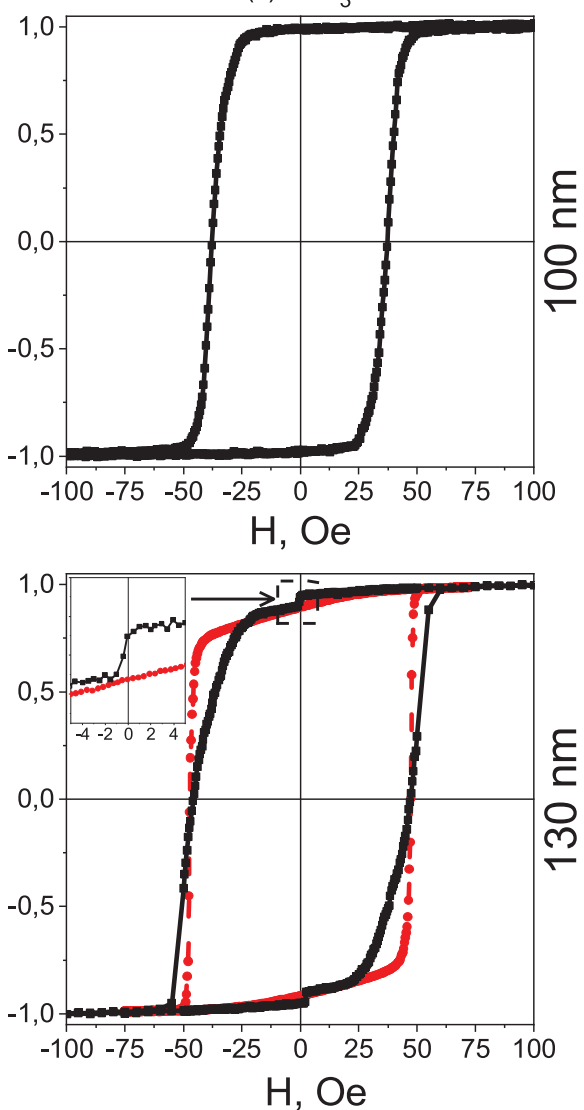

Fig. 2. (Colour online) Hysteresis loops along EMA for MPICs with different thicknesses of Py layer obtained by integral (black solid line with rectangles) and local (red dashed line with circles) methods. The first and second rows represent the hysteresis loops for MPICs with permalloy thicknesses of 100 and

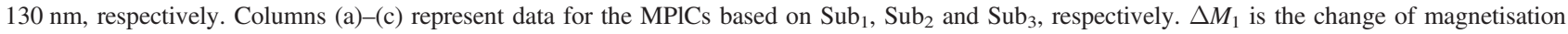
associated with the magnetisation vector rotation and $\Delta M_{2}$ is the change of magnetisation related to the domain wall propagation. 
(a) Sub 1
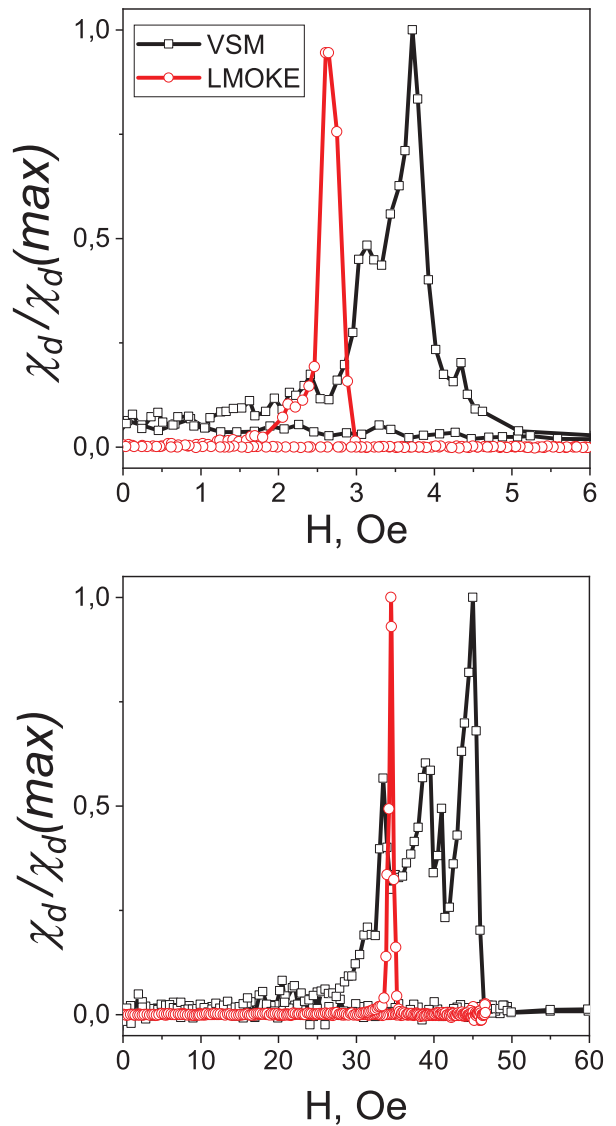

(b) $\mathrm{Sub}_{2}$
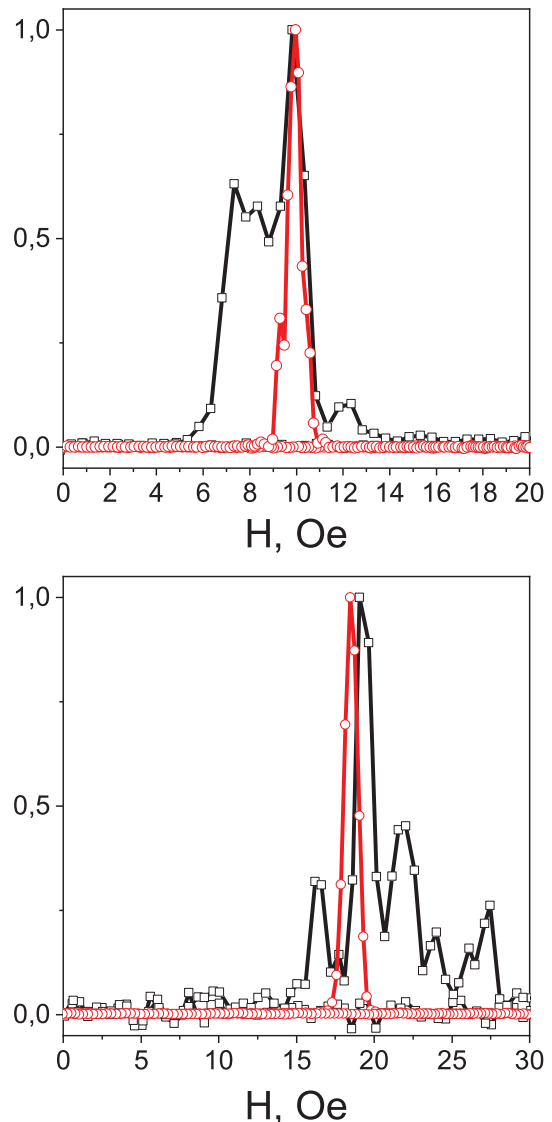

(c) $\mathrm{Sub}_{3}$
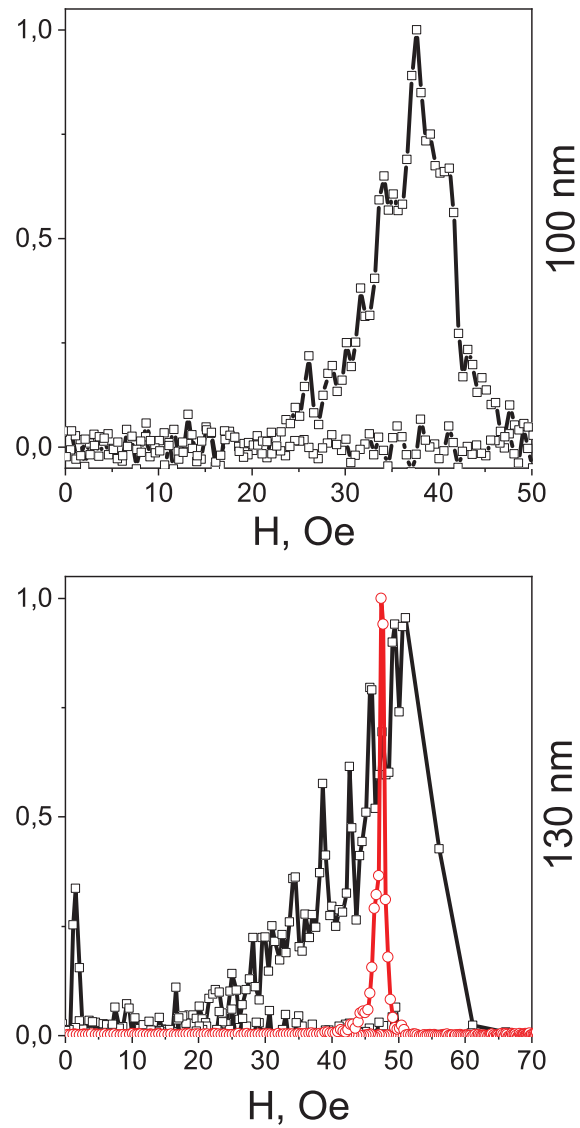

Fig. 3. (Colour online) Differential susceptibility normalised to the maximum $\chi_{d} / \chi_{d}$ (max) for MPICs with different Py thicknesses obtained by integral (black solid line with open rectangles) and local (red lines with open circles) methods. The first and the second rows correspond to the samples with permalloy layers of 100 and $130 \mathrm{~nm}$, respectively. Columns (a)-(c) represent data for the MPICs based on Sub , Sub $_{2}$ and Sub $_{3}$, respectively.

contributions of morphology to the magnetisation reversal of the MPICs. ${ }^{33)}$

\section{Results and discussion}

\subsection{Results}

The dependences of the magnetic moment normalised to a saturation value on the external magnetic field measured by local and integral methods are shown in Fig. 2. The methods used to measure the magnetic properties have a principal difference arising from their features: the VSM allows measurement of the magnetic moment of the whole volume of the sample, including defects at its edges caused by the fabrication process, ${ }^{28)}$ on the other hand, the LMOKE geometry allows examination of the local magnetic properties, in an area limited by the light penetration depth and the diameter of the light beam.

A rectangular hysteresis loop of the surface area means the magnetisation reversal was mainly driven by domain wall propagation in regions far from the edges. Magnetisation reversal proceeded additionally via rotation of the magnetisation vector and led to the tilting of the hysteresis loops in all cases for the VSM measurements. As a result, the integral hysteresis loops presented a smaller squareness ratio $Q=M_{\mathrm{r}} / M_{\mathrm{S}}\left(M_{\mathrm{r}}\right.$ being the remanent magnetisation and $M_{\mathrm{S}}$ the saturation magnetisation) than the local ones and the differential susceptibility calculated for the LMOKE loops had a narrower peak than that for the VSM. ${ }^{34,35)}$ The dependences of $\chi_{d}$ normalised to the maximum value on the magnetic field are shown in Fig. 3. The broadening of the SFD peaks for the VSM measurements (Fig. 3) may be connected with one of the following factors: (i) the polycrystalline nature of the permalloy films with a finite number of large grains of the peculates of the structure; (ii) magnetisation reversal processes of edge defects themselves; (iii) magnetic anisotropy fields having different magnitudes ef tilted and bent areas on the diffraction grating; and (iv) thickness-dependent mechanical stress in the permalloy layer.

The hysteresis loops measured with the VSM and LMOKE were only similar at fields around the coercive force $H_{\mathrm{C}}$ for each sample. Taking into account the ratio of the regions where the magnetisation was associated with the magnetisation vector rotation ( $\Delta M_{1}$ in Fig. 2) and was related to the domain wall propagation ( $\Delta M_{2}$ in Fig. 2), one can see that the main volume of the samples was magnetised through the domain wall propagation. The increase of in-plane $H_{\mathrm{C}}$ along the EMA with the permalloy layer thickness for both types of measurements is in agreement with theory and can be explained by the broadening of the classical Bloch wall with the increase of permalloy thickness from $100 \mathrm{~nm}$ to $130 \mathrm{~nm}^{33)}$

The positions of the $\chi_{d}$ peaks were almost the same for the VSM and LMOKE loops for the MPICs based on $\mathrm{Sub}_{2}$ and $\mathrm{Sub}_{3}$, while for the samples based on the smooth $\mathrm{Sub}_{1}$ substrates the $\chi_{d}$ peaks' positions shifted towards lower fields for the LMOKE curves. This can be explained by the existence of geometry-driven anisotropy dominating in the 
(a) In-plane

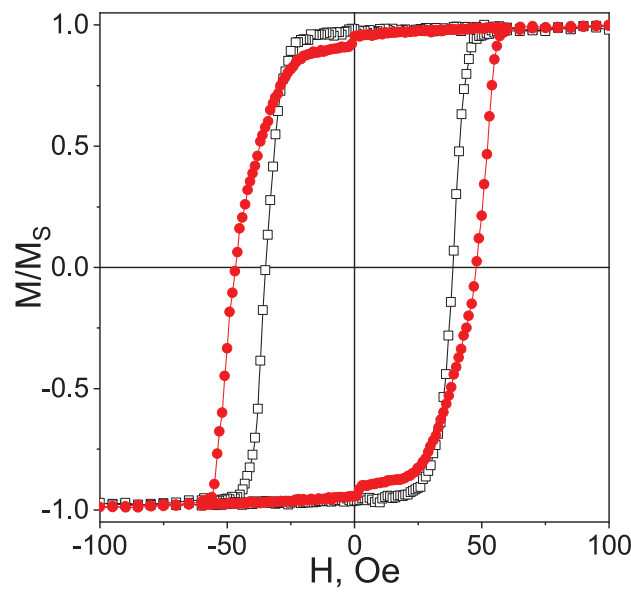

(c) Out-of-plane

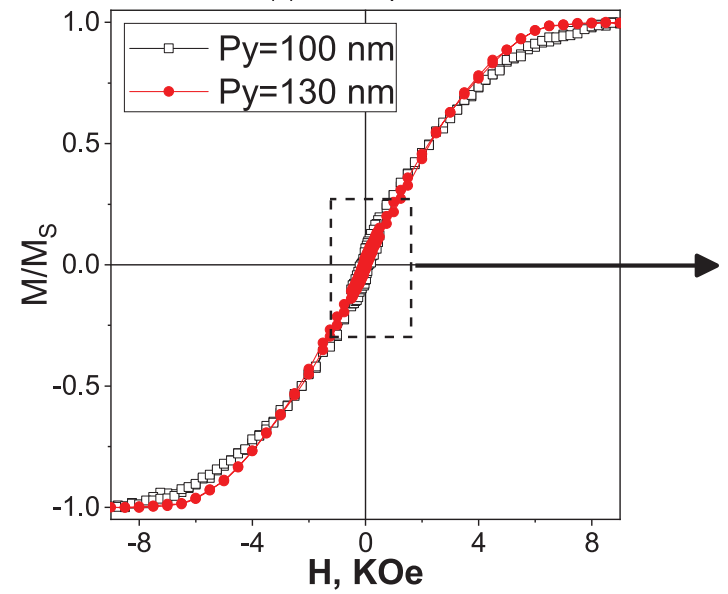

(b) At the angle of $45^{\circ}$

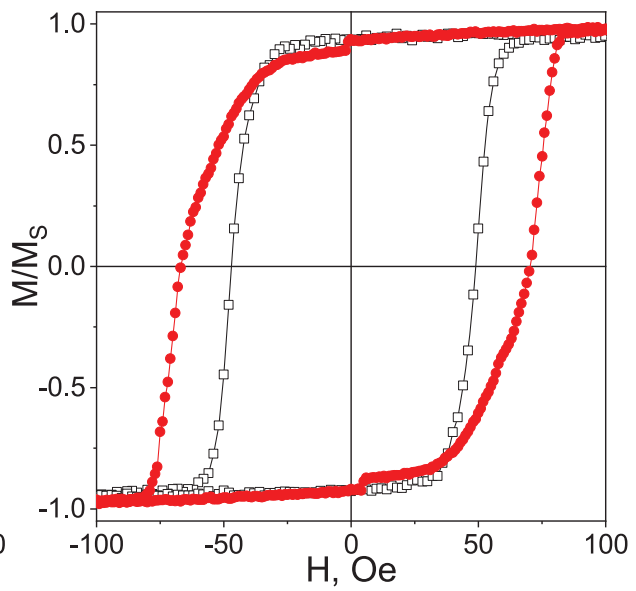

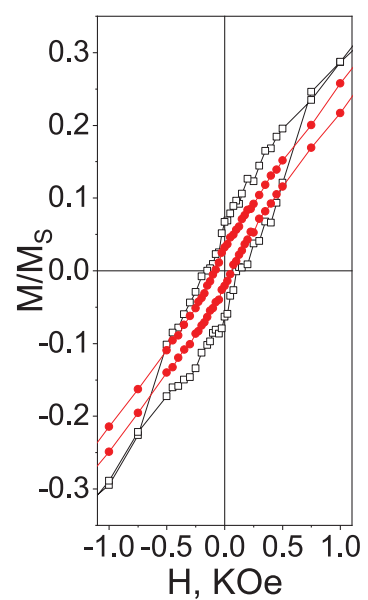

Fig. 4. (Colour online) Hysteresis loops of MPICs with permalloy thickness of $100 \mathrm{~nm}$ (black solid line with open rectangles) and $130 \mathrm{~nm}$ (red solid lines with solid circles) based on $\mathrm{Sub}_{3}$. Panels (a)-(c) correspond to the loops measured with the VSM in-plane along the EMA, under the angle of $45^{\circ}$ relative to the plane and in out-of-plane directions of the applied field, respectively.

MPICs and a stress effect through the thickness of the polycrystalline substrate-constrained films leading to the decrease of the anisotropy constant in the stress-free surface area.

Considering the VSM measurements for the smooth planar $\mathrm{Sub}_{1}$ based MPICs, additional peaks did not repeat in the magnitude, field position or number of ascending and descending parts of the hysteresis loops. This result is associated with the noise level of the system. On the other hand, the position for some peaks in the $\chi_{d}$ functions for MPICs based on the $\mathrm{Sub}_{2}$ and $\mathrm{Sub}_{3}$ substrates having morphological features repeated but the noise level also remained very high. The increase of permalloy layer thickness led to the heterogeneous behaviour of magnetic anisotropy and $\chi_{d}$, resulting in the appearance of additional peaks for the MPIC with a 130-nm-thick permalloy layer in comparison with MPICs based on thinner films. To determine the origin of all the peaks, additional structural and magnetic measurements should be done, for example, by the use of the first-order reversal curve (FORC) method. ${ }^{36,37)}$

In our previous work, devoted to the application of the considered MPICs as magnetic field sensors, it was shown that the required external modulation field and signal-to-noise ratio depend on the magnetic properties of the MPICs. ${ }^{26)}$ In particular, the required external modulation field is related to the coercive force, $H_{\mathrm{C}}$, and a measurable field region is determined by the slope of the signal-to-noise ratio function that has a phenomenological connection with the magnetisation curves. More information can be found in Ref. 26. The additional peaks at 46 Oe and 50 Oe seen in the $\chi_{d}$ function for the MPIC with a permalloy thickness of $130 \mathrm{~nm}$ based on $\mathrm{Sub}_{3}$ [Fig. 2(c) bottom, VSM] correspond to regions of steps on a step-like field dependence of the signal-to-noise ratio function. There was an ultra-soft repeatable magnetic phase with an $H_{\mathrm{C}}$ value of $1.5 \mathrm{Oe}$. This magnetic phase in the future could be used to measure external magnetic fields in very low modulating fields with magnitudes of less than 1 Oe. The contribution of the ultra-soft magnetic phase can be further increased by tuning the ferromagnetic layer thickness.

Additionally, the hysteresis loops for the $\mathrm{Sub}_{3}$-based MPICs were measured with a VSM in three directions of the external magnetic field. The measured loops are shown in Fig. 4. The sequential change of the magnetisation direction from in-plane along the EMA to an angle of $45^{\circ}$ relative to the plane, and then to the out-of-plane direction, led to the increase of $H_{\mathrm{C}}$. The mechanism of this effect is connected to the presence of some additional EMAs out of the sample's plane. They can be associated with morphological features that appeared with the changing of the magnetisation direction $^{32)}$ of the MPIC based on the $\mathrm{Sub}_{3}$ substrate.

To estimate the influence of the MPICs' morphology on the magnetisation reversal process the FWHM of the main 
Table I. FWHM of $\chi_{d} / \chi_{d}(\mathbf{m a x})$, measured by VSM and LMOKE techniques.

\begin{tabular}{|c|c|c|c|c|c|c|}
\hline \multirow{3}{*}{ Measurement technique } & \multicolumn{6}{|c|}{ Substrate//Thickness of Py, nm } \\
\hline & $\mathrm{Sub}_{1} / / 100$ & $\mathrm{Sub}_{1} / / 130$ & $\mathrm{Sub}_{2} / / 100$ & $\mathrm{Sub}_{2} / / 130$ & $\mathrm{Sub}_{3} / / 100$ & $\mathrm{Sub}_{3} / / 130$ \\
\hline & \multicolumn{6}{|c|}{ FWHM, Oe } \\
\hline VSM & 0.52 & 2.44 & 3.35 & 4.42 & 8.40 & 11.10 \\
\hline LMOKE & 0.24 & 0.6 & 0.87 & 1.02 & 0.69 & 0.82 \\
\hline
\end{tabular}

peaks of the $\chi_{d}$ functions was calculated and the results are presented in Table I.

The appearance of a profile with a sinusoidal or trapezoidal shape $\left(\mathrm{Sub}_{2}\right.$ or $\mathrm{Sub}_{3}$ substrate, respectively) was followed by the growth of $\chi_{d} / \chi_{d}(\max )$ width in comparison with the smooth $\mathrm{Sub}_{1}$ based samples. Changes in the permalloy layer thickness also resulted in the broadening of the FWHM regardless of the measurement method for each sample. For the integral VSM measurements, one can see that the increase of the $h$ and $d$ parameters led to the broadening of $\chi_{d} / \chi_{d}(\max )$ width related to the prevailing contribution of magnetisation reversal processes (i)-(iv). On the other hand, for the local LMOKE measurements there was a decrease of $\chi_{d} / \chi_{d}(\max )$ width with increasing $h$ and $d$ parameters. This is presumably related to the surface microstructure of the MPICs being determined by the features of the permalloy grains (depending on the MPIC profile shape) rather than by stress-related factors from the substrate. Broadening means that there were larger grains in the permalloy film $^{38)}$ considering the MPICs based on $\mathrm{Sub}_{2}$ in comparison with the $\mathrm{Sub}_{3}$-based MPICs.

In summary, we have established that the step-like behaviour of magneto-optical properties ${ }^{26)}$ correlates with the integral magnetic properties, but does not correlate with the local ones. This can be explained by the appearance of millimetre-scale features (region of surface for signal-tonoise ratio studies) and the absence of micron-scale features (LMOKE studies), which will be studied further.

\section{Conclusions}

The detailed measurements of magnetic properties provided in this paper show a way to tune the required modulation field and the measurable field region of a magnetic field sensor based on MPlCs. This can be done by changing the ferromagnetic layer thickness and the substrate's parameters influencing the magnetic and magneto-optical properties. It was confirmed that the origin of the multi-region MPIC-based sensor is the additional magnetic phases in the MPIC based on $\mathrm{Sub}_{3}$ with $130 \mathrm{~nm}$ permalloy. This makes it possible to create a magnetic field sensor working in two different regions of the measurable magnetic field. In future works, the contributions of the magnetisation vector rotation and domain wall propagation will be additionally studied by local and integral FORC diagrams in multiphase MPICs.

\section{Acknowledgments}

Discussion of the results has been made possible through a mobility grant provided by the 5 Top 100 Russian Academic Excellence Project at the Immanuel Kant Baltic Federal University. M. Rivas acknowledges the support of the Principality of Asturias under project IDI/2018/000185. V. V. Rodionova acknowledges financial support from the
Russian Ministry of Education and Science in the framework of Government Assignment 3.4168.2017/4.6.

\section{ORCID iDs}

D. V. Murzin (iD https://orcid.org/0000-0002-5180-8873

F. Groß (iD https://orcid.org/0000-0002-2412-285X

J. Gräfe (iD https://orcid.org/0000-0002-4597-5923

1) H. S. Park, J. S. Hwang, W. Y. Choi, D. S. Shim, K. W. Na, and S. O. Choi, Sens. Actuators A 114, 224 (2004).

2) L. Yan, B. Zhu, Z. Jiao, C.-Y. Chen, and I.-M. Chen, Sci. Rep. 4, 06756 (2014).

3) V. Markevicius, D. Navikas, M. Zilys, D. Andriukaitis, A. Valinevicius, and M. Cepenas, Sensors 16, 78 (2016).

4) J. Ng, J. Johnson, S. Miller, K. Newton, T. Roe, and R. Woo, Procedia CIRP 65, 180 (2017).

5) P. Ripka, J. Phys.: Conf. Ser. 450, 012001 (2013).

6) P. Novacek, J. Rohac, J. Simanek, and P. Ripka, IEEE Trans. Magn. 49, 69 (2013).

7) L. P. Ichkitidze, N. A. Bazaev, D. V. Telyshev, R. Y. Preobrazhensky, and M. L. Gavrushina, Biomed. Eng. 48, 305 (2015).

8) G. Lin., D. Makarov, and O. G. Schmidt, Lab Chip 17, 1884 (2017).

9) P. Brown et al., Rev. Sci. Instrum. 85, 125 (2014).

10) M. D. Michelena, I. Arruego, J. M. Other, and H. Guerrero, IEEE Trans. Aerospace Electron. Syst. 46, 542 (2010).

11) M. Paun, J. Sallese, and M. Kayal, J. Sens. Actuator Netw. 2, 85 (2013).

12) E. Ramsden, Hall Effect Sensors: Theory and Applications (Elsevier, Amsterdam, 2006) 2nd ed., p. 83.

13) R. L. Fagaly, Rev. Sci. Instrum. 77, 101101 (2006).

14) M. José Martínez-Pérez and D. Koelle, Phys. Sci. Rev. 2 (2017).

15) J. Gallop, Supercond. Sci. Technol. 16, 1575 (2003).

16) L. A. Francis and K. Poletkin, Magnetic Sensors and Devices: Technologies and Applications (CRC Press, London, 2018), p. 103.

17) A. I. Musorin, M. G. Barsukova, A. S. Shorokhov, B. S. Luk'yanchuk, and A. A. Fedyanin, J. Magn. Magn. Mater. 459, 165 (2018).

18) B. F. Diaz-Valencia, J. R. Mejía-Salazar, O. N. Oliveira Jr., N. PorrasMontenegro, and P. Albella, ACS Omega 2, 7682 (2017).

19) A. A. Grunin, I. R. Mukha, A. V. Chetvertukhin, and A. A. Fedyanin, J. Magn. Magn. Mater. 415, 72 (2016).

20) A. A. Grunin, A. G. Zhdanov, A. A. Ezhov, E. A. Ganshina, and A. A. Fedyanin, Appl. Phys. Lett. 97, 261908 (2010).

21) V. I. Belotelov, I. A. Akimov, M. Pohl, V. A. Kotov, S. Kasture, A. S. Vengurlekar, A. V. Gopal, D. R. Yakovlev, A. K. Zvezdin, and M. Bayer, Nat. Nanotechnol. 6, 370 (2011).

22) A. L. Chekhov, P. N. Naydenov, M. N. Smirnova, V. A. Ketsko, A. I. Stognij, and T. V. Murzina, Opt. Express 26, 21086 (2018).

23) V. Belyaev, A. Grunin, A. Fedyanin, and V. Rodionova, Solid State Phenom. 233-234, 599602 (2015).

24) A. V. Zayats and I. I. Smolyaninov, J. Opt. A 5, 1650 (2003).

25) L. Novotny and B. Hecht, Principles of Nano-Optics (Cambridge University Press, Cambridge, 2012) 2nd ed., p. 377.

26) V. K. Belyaev, D. V. Murzin, N. N. Perova, A. A. Grunin, A. A. Fedyanin, and V. V. Rodionova, J. Magn. Magn. Mater. 482, 292 (2019).

27) V. K. Belyaev, V. V. Rodionova, A. A. Grunin, M. Inoue, and A. A. Fedyanin, Sci. Rep. (2019).

28) V. K. Belyaev, A. G. Kozlov, A. V. Ognev, A. S. Samardak, and V. V. Rodionova, J. Magn. Magn. Mater. 480, 150 (2019).

29) A. G. Kozlov, M. E. Stebliy, A. V. Ognev, A. S. Samardak, A. V. Davydenko, and L. A. Chebotkevich, J. Magn. Magn. Mater. 422, 452 (2017).

30) V. Belyaev, A. Grunin, K. Chichay, S. Shevyratalov, A. Fedyanin, and V. Rodionova, Acta Phys. Pol. A 127, 546 (2015). 
31) S. N. Kim, Y. J. Nam, Y. D. Kim, J. W. Choi, H. Lee, and S. H. Lim, Sci. Rep. 6, 26709 (2016).

32) K. V. Yershov, V. P. Kravchuk, D. D. Sheka, and Y. Gaididei, Phys. Rev. B 92, 104412

33) J. C. Martínez-García, M. Rivas, D. Lago-Cachón, and J. A. García, J. Phys. D 47, 015001 (2013).

34) R. Schäfer, Magnets, Soft and Hard: Domains in Encyclopedia of Materials: Science and Technology (Elsevier, Amsterdam, 2001) 5130.
35) M. Rivas, P. Gorria, C. Munoz-Gomez, and J. C. Martinez-Garcia, IEEE Trans. Magn. 53, 7907161 (2017).

36) M. Rivas, J. C. Martínez-García, I. Škorvánek, J. Marcin, P. Švec, and P. Gorria, Appl. Phys. Lett. 107, 132403 (2015)

37) J. Gräfe, M. Weigand, N. Träger, G. Schütz, and E. J. Goering, Phys. Rev. B 93, 104421 (2016).

38) M. D. Graef, Magnetic Imaging and Its Applications to Materials (Academic, San Diego, 2000), p. 109. 